\title{
Sliding Mode Control with RBF Neural Network for Two Link Robot Manipulator
}

\author{
Ankita Yadav \\ Department of Electrical \\ Engineering \\ Delhi Technological University
}

\author{
Ajit Kumar Sharma \\ Department of Electrical \\ Engineering \\ Delhi Technological University
}

\author{
Bharat Bhushan \\ Department of Electrical \\ Engineering \\ Delhi Technological University
}

\begin{abstract}
Nonlinear control techniques are applied on mechanical systems namely two link robot manipulator to study the effect of the controllers on the tracking performance of the two system. A design of sliding mode control(SMC) for the position tracking of two link robot manipulator based on the sliding mode control technique and the Lyapunov stability theory is carried out to eliminate the perturbation and asymptotical stability can be achieved when the system is subjected to the sliding mode. A sliding mode control method based on $\mathrm{RBF}$ (radial basis function) neural network is addressed which has the capability of learning uncertain control actions shown by the several industrial robots. In RBFNN-SMC method the algorithm for tuning the parameters are extracted from the RBF function. The comparative study is done based on the evaluated parameters for the system
\end{abstract}

\section{Keywords}

Sliding mode control, RBF Neural Network, Two-link robot manipulator.

\section{INTRODUCTION}

Sliding mode control has been the vast topic for research in the field of control engineering. This section discuss Extensive research work has been done on sliding mode control of the system as well as the integration of compensation technique with this control methodology.[1] It is shown that the adaptive sliding mode control to control a 2 link robot manipulator having uncertain parameters. An adaptive algorithm is used which is based on the sliding mode control concept to ease the chattering phenomena. Lyapunov stability is applied to achieve the stability of robot manipulator system. Controller scheme proposed in that assures robustness and attain good trajectory tracking performance.[2] Conventional design methods for controllers for a MIMO plant like a multi-joint robot require minimum knowledge of the structure and accurate mathematical model of the plant. [3] The dynamic model and the terminal sliding mode control for two link robot manipulators. Lagrangian method is used to derive the closed loop dynamic equations for the robot manipulators. This technique has been used to design the controller. This technique gives the output tracking error to converge at zero in a given period of time. [4] A radial basis function network with SMC is designed for the link positions of two link robot manipulator. The learning techniques has degrades its transient performance also This neural technique uses the curve fitting mode to get nonlinear mapping. A sliding mode effectively used to eliminate thenonlinearities and has given a fast response but this control created chattering. The Lyapunov and the back propagation algorithm creates switch gain and an update law respectively.[5] Computed torque control is one of such schemes, which is based on the exact cancellation of the nonlinear dynamics of the manipulator system.[6] ]Robot manipulators are very important in the field of flexible automation as they have been widely used for increasing productivity and reduce losses. A lot of research effort is been made to design their controller.[7] ]Robot manipulators are very important in the field of flexible automation as they have been widely used for increasing productivity and reduce losses. A lot of research effort is been made to design their controller.[8] Neural Networks are being applied in many fields such as learning, pattern recognition, signal processing and system control. The major advantages this parallel structure, learning ability, non- linear function approximation, fault tolerance. This benefits make a wide range of applications..[9] Robot manipulators are very important in the field of flexible automation as they have been widely used for increasing productivity and reduce losses. A lot of research effort is been made to design their controller.[10] The payload of the robot manipulator varies during its operation and is not known advance. To face such problems, adaptive control strategies for robot manipulators have been developed.[11] Research from the past shows that universal approximation theorems on RBF has shown that any nonlinear function over a compact set with arbitrary accuracy can be approximated by RBF neural network.[12] The RBF network adaptation improves the control performance against large uncertainty of the system. The adaptation law can be derived by Lyapunov method which guarantees the stability of the system. RBF neural networks. A schematic diagram of two link robot manipulator is shown in figure 1 .

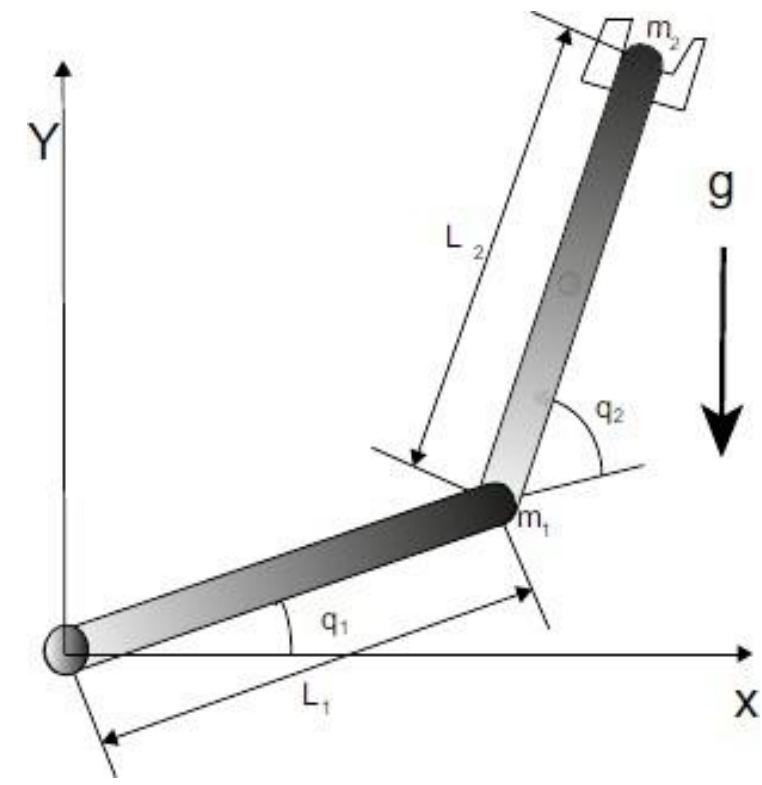

Figure 1: A two link robot manipulator 


\section{SLIDING MODE CONTROL}

Sliding mode control deals with the problem of model uncertainties as these uncertainties can have strong adverse effects on nonlinear control systems. A major approach to deal with the model uncertainly is adaptive control and another approaches which can be used to solve the control problems includes the sliding mode techniques. These techniques are generating greater interest nowadays. Discrepancies may occur between the actual plant and the mathematical model established for the controller design. Various factors may be responsible for this mismatch. The engineer's role is to ensure required performance levels for the system instead of such mismatches. A set of robust control methods have been developed to eliminate any error. One such method is sliding mode control methodology(SMC). This is a specific type of variable structure control system (VSCS). SMC has been used for several systems including nonlinear system, multi-input multi- output(MIMO) systems, discrete-time models, large-scale and infinite-dimension systems, and stochastic systems. SMC is completely insensitive to parametric imprecisions and external disturbances during sliding mode. VSC uses a high-speed switching control law to accomplish two objectives. Firstly, the nonlinear plant's state trajectory is taken onto a specified and user-chosen surface in the state space which is called the sliding or switching surface. This is called as the switching surface because a control path has a unique gain if the state trajectory of the plant is "above" the surface and a different gain if the trajectory falls "below" the surface. Secondly, it keeps the plant's state trajectory on this surface for all consequent times. During this process, the control system's structure changes from one to another and thus given the name variable structure control(VSC). The control is also named as the sliding mode control to accentuate the importance of the sliding mode. Sliding mode controller can stabilize the trajectory of a system. Control structures are designed so as to ensure that trajectories will always move towards a switching condition. Therefore, the ultimate trajectory will not exist completely within one control structure. Instead, the ultimate trajectory will slide along the boundaries of the control structures. The motion of the system as it slides along these boundaries is termed as a sliding mode and the geometrical locus consisting of the boundaries is called the sliding (hyper) surface. The sliding mode phases are shown in figure 2

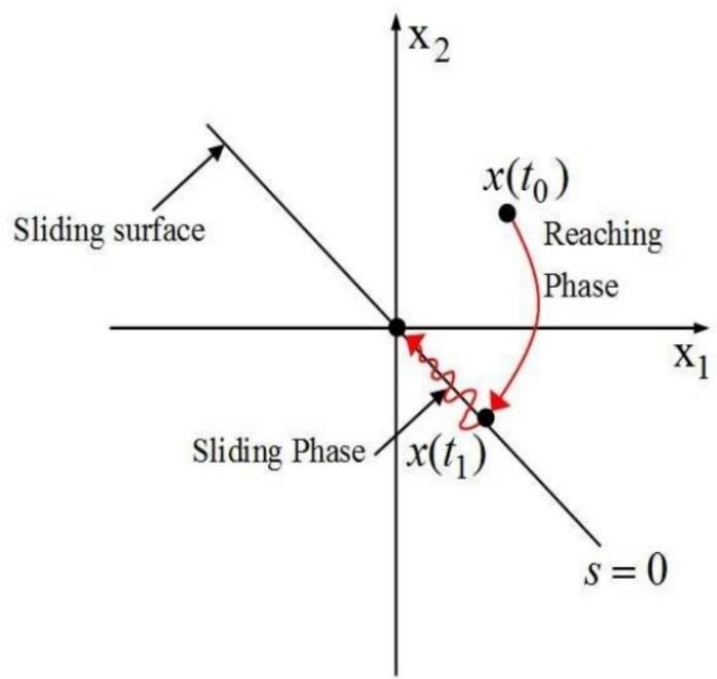

Figure 2: Sliding mode phases

\section{LYAPUNOV STABILITY}

The Lyapunov stability analysis is based upon the concept of energy and also depends on the relation of stored energy with the stability of system. But in general there is no way of relating the energy function with the given set of equations which describes the system. There is no uniqueness about the total energy of the system which associates with the system stability. There are scalar functions present which describe this relation and this ides is given by the mathematician A.M Lyapunov. The scalar functions are known as the Lyapunov Function and the method used for evaluating the stability of the control system is known as the Lyapunov's direct method. Following are some of the scalar functions which are described as the follows by the Lyapunov direct method. Based on these functions, stability can be evaluated.

(a)Positive Definiteness of Scalar Functions : V(x) which is a scalar function is said to be positive definite in the region $\|\mathrm{x}\| \leq \mathrm{k}$ if $\mathrm{V}(\mathrm{x})>0$ at all the points of the region except origin since it is zero at origin.

(b)Negative Definiteness of Scalar Functions: V(x) which is a scalar function is said to be negative definite if $[-\mathrm{V}(\mathrm{x})]$ is positive definite.

(c)Positive Semi definiteness of Scalar Functions: V(x) which is a scalar function is said to be positive semidefinite in the region $\|\mathrm{x}\| \leq \mathrm{k}$ if its value is positive at all points of the region and at origin too.

(d)Negative Semi definiteness of Scalar Functions: V(x) which is a scalar function is said to be negative definite if $[-\mathrm{V}(\mathrm{x})]$ is positive semidefinite.

\section{RBF NEURAL NETWORK}

This is an alternative approach for multilayer perceptron which is trained by the backpropagation algorithm. It is the basic component of feedforward network. A gaussian function has two parameters: center for defining its position and for determining its shape, parameter called spread is there. In one dimensional gaussian function spread is same as the standard deviation $\sigma$.

The input vector is given by

$x=\left[\begin{array}{lllll}x_{1} & x_{2} & \ldots & \ldots & \ldots \\ x_{n}\end{array}\right]$

and the output of the RBF network is given below

$\mathrm{x}(\mathrm{x}, \mathrm{c}, \sigma)=\exp \frac{-\|x-c\|^{2}}{2 \sigma^{2}}$

there is no weights between the input and the RBF unit. $\mathrm{c}$ and $\sigma$ represents the weight which is shown in figure 3 . 


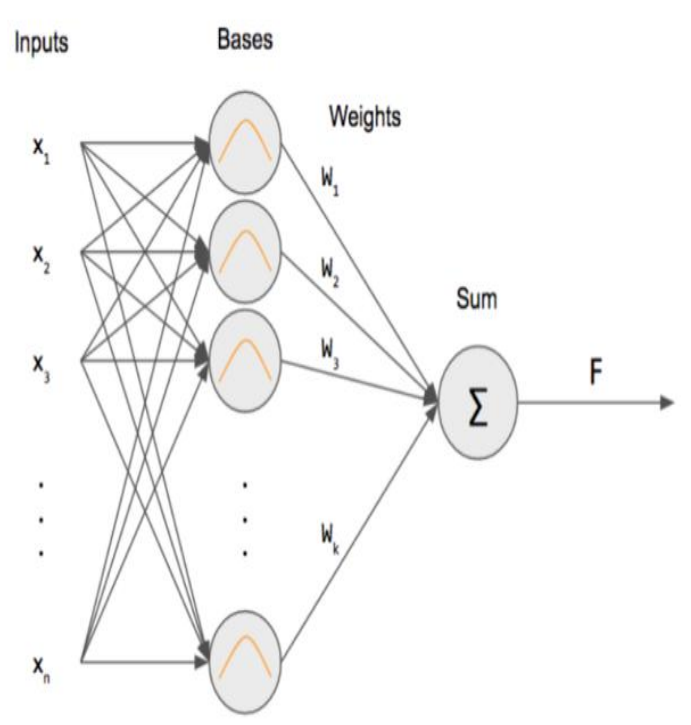

Figure 3: Gaussian function in RBF neuron model

\section{SLIDING MODE CONTROLLER FOR A TWO LINK ROBOT}

The plant model is given by equation (3)

$$
\begin{gathered}
\dot{x_{1}}=\dot{\theta}=x_{2} \\
\dot{x_{2}}=\ddot{\theta}=f(x)+g(x) \tau
\end{gathered}
$$

Where

$$
x=\left[\begin{array}{l}
x_{1} \\
x_{2}
\end{array}\right]=\left[\begin{array}{l}
x_{11} \\
x_{12} \\
x_{21} \\
x_{22}
\end{array}\right]=\left[\begin{array}{l}
\theta \\
\dot{\theta}
\end{array}\right]=\left[\begin{array}{c}
\theta_{1} \\
\theta_{2} \\
\dot{\theta}_{1} \\
\dot{\theta}_{2}
\end{array}\right] ; \tau=\left[\begin{array}{l}
\tau_{1} \\
\tau_{2}
\end{array}\right]
$$

$\theta_{1}$ and $\theta_{2}$ are the angles of the two links defined in figure, and $\tau_{1}$ and $\tau_{2}$ are the torques applied by the actuators to control the angles $\theta_{1}$ and $\theta_{2}$ respectively.

Variable structure sliding mode controller design consists of the following two phases:

(a)Designing of sliding surface to obtain the desired system behaviour, when restricted to the surface. The system will remain at that surface for further times. The plant dynamics will always be limited to the surface and is robust towards the disturbances.

(b)Selection of the feedback gains of the controller, so that the closed loop system is stable to the sliding surface.

For a two link robot manipulator, our aim is to track the desired motion trajectory $\theta_{d}(t)$

The tracking error is defined a

$$
\begin{gathered}
e(t)=\theta_{d}(t)-\theta(t) \\
\dot{e(t)}=\dot{\theta_{d}}(t)-\dot{\theta}(t) ; \ddot{e}(t)=\ddot{\theta_{d}}(t)-\ddot{\theta}(t)
\end{gathered}
$$

Defining $\widetilde{x_{1}}=e$ and $\widetilde{x_{2}}=\dot{e}$ robot dynamics can be written in the form:

$\dot{x_{2}}=\ddot{\theta}=f(x)+g(x) \tau$

Now we use linear sliding surface which is defined by the equation

$\sigma(\tilde{x})=\lambda \widetilde{x_{1}}+I \widetilde{x_{2}}$
Or

$$
\begin{aligned}
& {\left[\begin{array}{l}
\sigma_{1}(\tilde{x}) \\
\sigma_{2}(\tilde{x})
\end{array}\right]=\left[\begin{array}{cc}
\lambda_{1} & 0 \\
0 & \lambda_{2}
\end{array}\right]\left[\begin{array}{l}
\widetilde{x_{11}} \\
\widetilde{x_{12}}
\end{array}\right]+\left[\begin{array}{ll}
1 & 0 \\
0 & 1
\end{array}\right]\left[\begin{array}{l}
\widetilde{x_{21}} \\
\widetilde{x_{22}}
\end{array}\right]=\left[\begin{array}{l}
0 \\
0
\end{array}\right](7)} \\
& \sigma_{1}(\tilde{x})=\lambda_{1} \tilde{x}_{11}+\widetilde{x_{21}}=\lambda_{1}\left(\theta_{1 d}-\theta_{1}\right)+\left(\dot{\theta_{1 d}}-\dot{\theta}_{1}\right) \\
& \sigma_{2}(\tilde{x})=\lambda_{2} \tilde{x}_{12}+\widetilde{x_{22}}=\lambda_{2}\left(\theta_{2 d}-\theta_{2}\right)+\left(\dot{\theta_{2 d}}-\dot{\theta}_{2}\right)
\end{aligned}
$$

Assume the coefficient of $\widetilde{x_{21}}$ to be unity.

Now combine the equations of the plant and the sliding surface (Eqns (7) and (8))

$\dot{\tilde{x_{1}}}=-\lambda \widetilde{x_{1}}$

Therefore

$$
\widetilde{x_{2}}=-\lambda \tilde{x}_{1}
$$

The above equations describes the system dynamics in sliding. The response is specified by the selection of the parameters $\lambda_{1}$ and $\lambda_{2}$ of the switching surface. In sliding mode, the system is not affected by the model uncertainties. After designing the sliding surface, we build a feedback controller. The objective of the controller is to derive the plant state to the sliding surface and maintain it on the surface for further time. A Lyapunov approach has been used for building the controller.

The Lyapunov function is given below

$V=\frac{1}{2} \sigma^{T} \sigma=\frac{1}{2}\left(\sigma_{1}^{2}+\sigma_{2}^{2}\right)$

The above function is used as the Lyapunov function and the time derivative of the chosen Lyapunov function comes out to be negative definite with respect to switching surface and ensuring the motion of the state trajectory to the surface. We have to find $\tau$ so that

$$
\begin{gathered}
\frac{d}{d t} \frac{1}{2} \sigma^{T} \sigma=\sigma^{T} \dot{\sigma}<0 \\
\sigma^{T} \dot{\sigma}=\sigma^{T}\left[\lambda \dot{\widetilde{x_{1}}}+I \dot{\widetilde{x_{2}}}\right]
\end{gathered}
$$

The controller structure form is given below

$$
\tau=g^{-1}(x)\left[-f(x)+\dot{x}_{2 d}+\lambda\left(x_{1 d}-\dot{x_{1}}\right)+\left\{\begin{array}{l}
k_{1} \operatorname{sgn}\left(\sigma_{1}\right) \\
k_{2} \operatorname{sgn}\left(\sigma_{2}\right)
\end{array}\right\}\right]
$$

where $k_{1}>0$ and $k_{2}>0$ are the gains which has to be determined so that the condition $\sigma^{T} \dot{\sigma}<0$ is satisfied. We substitute $\tau$, given by (3.52) into the expression $\sigma^{T} \dot{\sigma}$

$$
\begin{aligned}
& \sigma^{T} \dot{\sigma}=-\left[\begin{array}{ll}
\sigma_{1} & \sigma_{2}
\end{array}\right]\left[\begin{array}{l}
k_{1} \operatorname{sgn}\left(\sigma_{1}\right) \\
k_{2} \operatorname{sgn}\left(\sigma_{2}\right)
\end{array}\right]=-\sigma_{1} k_{1} \operatorname{sgn}\left(\sigma_{1}\right)- \\
& \sigma_{2} k_{2} \operatorname{sgn}\left(\sigma_{2}\right)=-k_{1}\left|\sigma_{1}\right|-k_{2}\left|\sigma_{2}\right|<0
\end{aligned}
$$

The sliding surface $\sigma(\tilde{x})=0$ is therefore asymptotically attractive. The larger the value of gains, faster the trajectory converges to the sliding surface. The tolerance of the sliding mode control to the model imprecision and disturbances, is high and thus satisfying stability requirement. The Simulink model for the sliding mode control of the two link robot manipulator is given in figure 3.2

Simulation of this controller for a two link robot manipulator was done in MATLAB and results are attached at the end.

The specifications of the robot manipulator is taken as: 
$m_{1}=1 \mathrm{~kg} ; m_{2}=1 \mathrm{~kg} ; l_{1}=1 \mathrm{~m} ; l_{2}=1 \mathrm{~m} ; g=$

$\frac{9.8 \mathrm{kgm}}{s^{2}} ; \theta_{d 1}(t)=\sin (\pi t) ; \theta_{d 2}(t)=\cos (\pi t)$

Now the objective is to construct an RBF network sliding mode control for robot manipulator. This is completely opposite to the SMC technique so far used as the unknow disturbances are approximated by RBF neural network.

\section{RBF NETWORK ADAPTIVE SLIDING MODE CONTROL FOR ROBOT MANIPULATOR}

The dynamic equation of 2-joint manipulator is as follows:

$$
H(q) \ddot{q}+C(q, \dot{q}) \dot{q}+G(q)=\tau-F(\dot{q})-\tau_{d}
$$

where $\mathrm{H}(\mathrm{q})$ is a $2 \times 2$ positive definite integral matrix, $\mathrm{G}(\mathrm{q})$ is a $2 \times 1$ inertial vector, $C(q, q)$ is a $2 \times 2$ inertial matrix, $\tau_{d}$ is the unknown disturbance, $F(\dot{q})$ is friction force, and $\tau$ is the control input

The tracking error is denoted as:

$e(t)=q_{d}(t)-q(t)$

Sliding variable is selected as:

$$
s=\dot{e}+\dot{\Lambda} e
$$

where $\Lambda$ is a symmetric positive definite matrix and $\Lambda=$ $\Lambda^{T}>0$, therefore, we have

$$
\begin{gathered}
\dot{q}=-s+\dot{q_{d}}+\Lambda e \\
H \dot{s}=H\left(\ddot{q_{d}}-\ddot{q}+\Lambda \dot{e}\right)=H\left(\ddot{q_{d}}+\Lambda \dot{e}\right)-H \ddot{q} \\
=H\left(\ddot{q_{d}}+\Lambda \dot{e}\right)-C s+C\left(\dot{q_{d}}+\Lambda e\right)+G+F+\tau_{d}-\tau \\
=H\left(\ddot{q_{d}}+\Lambda \dot{e}\right)+C \dot{q}+G+F+\tau_{d}-\tau \\
=-C s-\tau+f+\tau_{d}
\end{gathered}
$$

Where $\mathrm{f}(\mathrm{x})=H\left(\ddot{q_{d}}+\Lambda \dot{e}\right)+C\left(q_{d}+\Lambda e\right)+G+F$

as $f(x)$ is unknown, hence RBF network is adopted to approximate $f(x)$. input is selected based on expression of $f(x)$ :

$x=\left[\begin{array}{lllll}e^{T} & \dot{e}^{T} & q_{d}^{T} & \dot{q}_{d}^{T} & \ddot{q}_{d}^{T}\end{array}\right]$

The controller is designed as follows:

$$
\tau=\hat{f}(x)+K_{v} s
$$

where $\mathrm{Kv}$ is a symmetrical positive definite constant matrix, $\hat{f}(x)$ is the output of RBF

network, approximates $\mathrm{f}(\mathrm{x})$. The RBF-NN weights are adjusted using adaptive laws

From equations (21) and (22) we have

$$
H \dot{s}=-C s-\hat{f}(x)-K v s+f(x)+\tau_{d}
$$

Where

$$
f(x)=-\left(K_{v}+C\right) s+\tilde{f}(x)+\tau_{d}=-\left(K_{v}+C\right) s+\varsigma_{0}
$$

Lyapunov function is selected as:

$L=\frac{1}{2} s^{T} H s$

$\dot{L}=s^{T} H s+\frac{1}{2} s^{T} H=-s^{T} K_{v} s+\frac{1}{2} s^{T}(H-2 C) s+s^{T} S_{0}$

$L=s^{T} S_{0}-s^{T} K_{v} s$
It shows that with $\mathrm{Kv}$, the stability of control system depends on $\varsigma_{0}$, the magnitude of $\tau_{d}$

The algorithm suggested to approximate $\mathrm{f}(\mathrm{x})$ is given below

$$
\begin{aligned}
& \phi_{i}=g\left(\frac{\left\|x-c_{i}\right\|^{2}}{\sigma_{i}^{2}}\right) \\
& y=W^{* T} \varphi(x), f(x)=W^{* T} \varphi(x)+\varepsilon
\end{aligned}
$$

Where $\mathrm{x}$ is the input state of network,

$$
\varphi(x)=\left[\begin{array}{llll}
\phi_{1} & \phi_{2} & \ldots \ldots & \phi_{n}
\end{array}\right]^{T}
$$

$\varepsilon$ is the approximation error, $\mathrm{W}^{*}$ is the desired weight vector.

\section{SIMULATION AND RESULTS}

Kinetic equation of two joint robot manipulator is:

$H(q) \ddot{q}+C(q, \dot{q}) \dot{q}+G(q)=\tau-F(\dot{q})-\tau_{d}$

Where

$$
\begin{aligned}
& H(q)=\left[\begin{array}{cc}
p_{1}+p_{2}+2 p_{3} \cos q_{2} & p_{2}+p_{3} \cos q_{2} \\
p_{2}+2 p_{3} \cos q_{2} & p_{2}
\end{array}\right] \\
& C(q, \dot{q})=\left[\begin{array}{cc}
-p_{3} q_{2} \sin q_{2} & -p_{3}\left(\dot{q}_{1}+\dot{q}_{2}\right) \sin q_{2} \\
p_{3} \dot{q}_{1} \sin q_{2} & 0
\end{array}\right] \\
& G(q)=\left[\begin{array}{cc}
p_{4} g \cos q_{1} & p_{5} g \cos \left(q_{1}+q_{2}\right) \\
p_{5} g \cos \left(q_{1}+q_{2}\right)
\end{array}\right] \\
& F(\dot{q})=0.02 \operatorname{sgn}(\dot{q}) ; \tau_{d}=\left[\begin{array}{ll}
0.2 \sin t & 0.2 \sin t
\end{array}\right]^{T}
\end{aligned}
$$

Let

$$
\begin{aligned}
\mathrm{p}=\left[\begin{array}{lllll}
p_{1} & p_{2} & p_{3} & p_{4} & p_{5}
\end{array}\right] \\
=\left[\begin{array}{lllll}
2.9 & 0.76 & 0.87 & 3.04
\end{array}\right.
\end{aligned}
$$

Gaussian function of the RBF network is selected to control the neural network. If the parameter is not suitable then mapping of the gauss function cannot be obtained and the RBF network is unavailable. The model for this is given in the figure 4 .

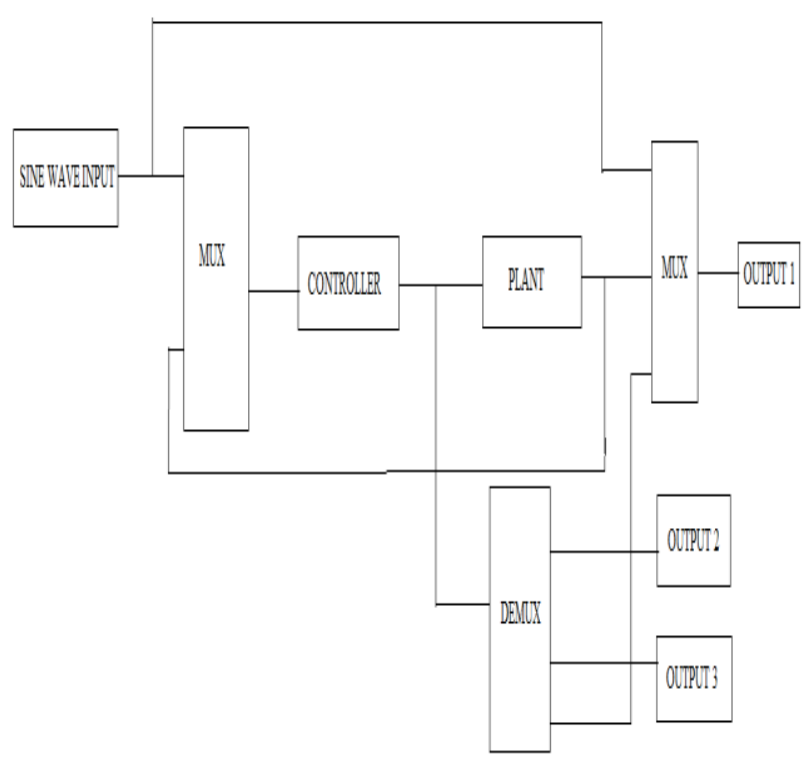

Figure 4: Block diagram for RBF network sliding mode control for two link robot manipulator 


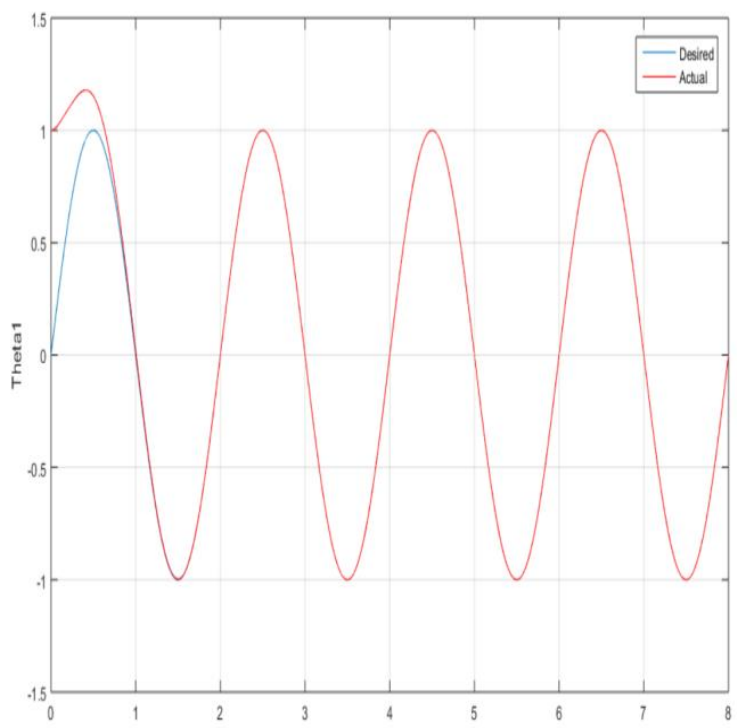

Figure 5: Desired and actual trajectory for link $1\left(\theta_{1}\right)$ using SMC

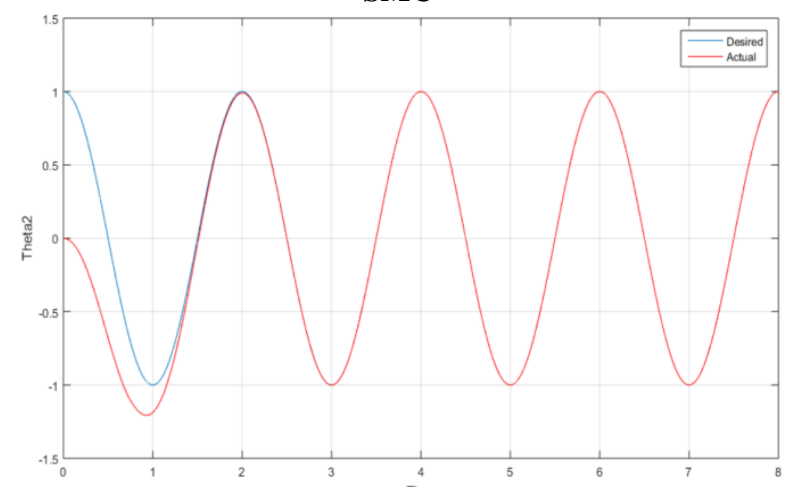

Figure 6: Desired and actual trajectory for link $2\left(\theta_{2}\right)$ using SMC

Table 1. Parameters for position of link 1 and 2

\begin{tabular}{|c|c|c|c|c|c|}
\hline LINKS & & $\begin{array}{c}\text { DELAY } \\
\text { TIME(sec) }\end{array}$ & $\begin{array}{l}\text { RISE TIME } \\
(\mathrm{sec})\end{array}$ & $\begin{array}{c}\text { PEAK TIME } \\
(\mathrm{sec})\end{array}$ & $\begin{array}{c}\text { PEAK OVERSHOOT } \\
\%\end{array}$ \\
\hline \multirow[t]{2}{*}{ LINK 1} & ACTUAL & 0.02134 & 0.1524 & 0.4113 & 17.9 \\
\hline & DESIRED & 0.03665 & 0.3618 & 0.5031 & 0 \\
\hline \multirow[t]{2}{*}{ LINK 2} & ACTUAL & 0.1969 & 0.7317 & 0.9231 & 14.53 \\
\hline & DESIRED & 0.2018 & 0.8579 & 0.9978 & 0 \\
\hline
\end{tabular}

Here figure 5 and 6 shows the desired and actual trajectory of the link 1 and link 2 of two link robot manipulator applied with SMC whereas figure 7 and 8 displays the same for RBFSMC

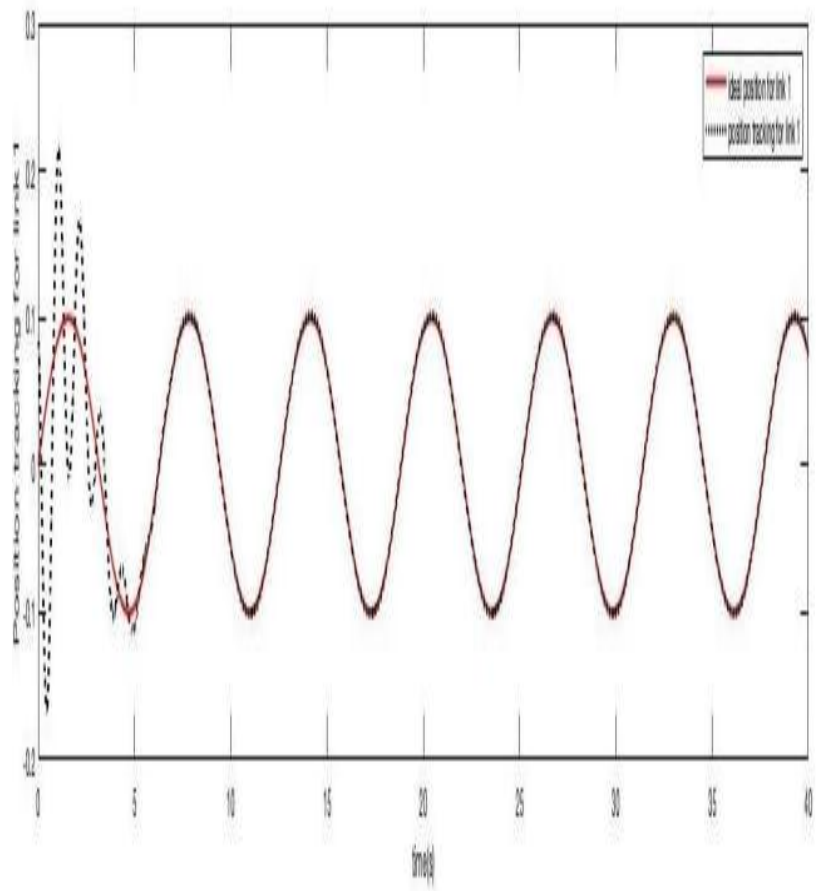

Figure 7: Desired and actual trajectory for link $1\left(\theta_{1}\right)$ using RBFNN-SMC 


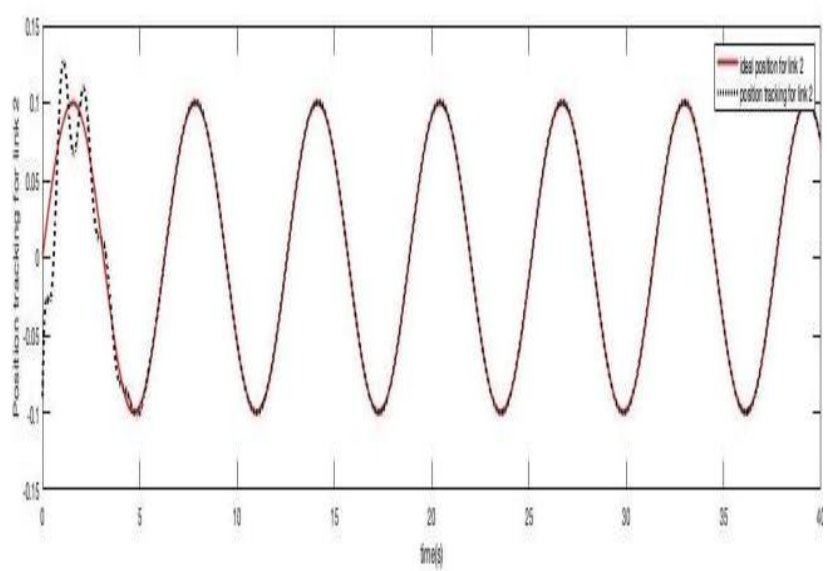

Figure 8: Desired and actual trajectory for link $2\left(\theta_{2}\right)$ using RBFNN-SMC

Table 2. Parameters for position of link 1 and 2

\begin{tabular}{|c|c|c|c|c|c|}
\hline LINKS & & $\begin{array}{c}\text { DELAY } \\
\text { TIME(sec) }\end{array}$ & $\begin{array}{l}\text { RISE TIME } \\
(\mathrm{sec})\end{array}$ & $\begin{array}{c}\text { PEAK TIME } \\
(\mathrm{sec})\end{array}$ & $\begin{array}{c}\text { PEAK OVERSHOOT } \\
\%\end{array}$ \\
\hline \multirow[t]{2}{*}{ LINK 1} & ACTUAL & 0.01874 & 0.1224 & 0.3216 & 14.18 \\
\hline & DESIRED & 0.02265 & 0.3103 & 0.3031 & 0 \\
\hline \multirow[t]{2}{*}{ LINK 2} & ACTUAL & 0.1472 & 0.6283 & 0.8163 & 12.44 \\
\hline & DESIRED & 0.1524 & 0.7152 & 0.8816 & 0 \\
\hline
\end{tabular}

\section{REFERENCES}

[1] K.M.Koo, J.H.Kim: Robust Control of Robot Manipulators with Parameters Uncertainty, IEEE Trans.Auto.Contr., Vol.39, No.6, 1230-1233,1994

[2] Utkin V.I., "Variable structure systems with sliding modes", IEEE Transactions on Automatic Control, vol. 22, no. 2, pp. 212-22, 1977.

[3] V. I. Utkin, Sliding Modes and Their Application in Variable Structure Systems. Moscow: MIR, 1978.

[4] Slotine J. and Sastry S., "Tracking control of nonlinear system using sliding surfaces with application to robot manipulators", International Journal of Control, vol. 38, pp.465-492, 1983.

[5] J.J.E.Slotine, W. Li: On the Adaptive Control of Robot Manipu -lators, The Int. J. Robotics Reseach,Vol.6,No.3, $49-59,1987$

[6] Slotine J.E. and Li W.P., "On the adaptive control of robot manipula-tors", Interational Journal of Robotics Research, vol. 6, no. 3, pp.49-59, 1987

[7] J.J.E.Slotine, W. Li: Adaptive Manipulators Control: A Case Study, IEEE Trans. Auto. Contr.,Vol.33,No.11,995 $-1003,1988$

[8] R. A. DeCarlo, S. H. Zak, and G. P. Matthews, "Variable Structure Control of Nonlinear Multivariate Systems: A

\section{CONCLUSIONS}

The mathematical model of two DOF robot manipulator is designed in the form of differential equations and then feedback linearization technique is applied to obtain the plant model and two control techniques that is adaptive sliding mode control and RBF neural network based sliding mode control have been successfully applied to improve the stability of this system.it is seen that position tracking is instantly and correctly achieved in case of RBF-SMC model, also the system implemented with RBF-SMC is more stable. A comparative study of the parameters for the links of robot manipulator has been carried out.
Tutorial," Proceedings of the IEEE, Vol. 76, No. 3, pp. 212-232, March 1988

[9] Astrom K.J. and Wittenmark B., "Adaptive Control", 2nd edn. Addison-Wesley, NY 1994,1989.

[10] V. I. Utkin and S. Drakunov, "On Discrete-time Sliding Mode Control," Proceedings of IFAC Symposium on Nonlinear Control Systems (NOLCOS), (Capri, Italy), pp. 484-489, 1989.

[11] Hartman EJ, Keeler JD, Kowalski JM. Layered neural networks with Gaussian hidden units as universal approximations. Neural computation, 1990, 2(2): 210 _ 215

[12] Kanellakopoulos I, Kokotovic PV, Morse AS (1991) Systematic design of adaptive controllersfor feedback linearizable systems. IEEE Trans Autom Control 36(11):1241-1253

[13] Park J, Sandberg IW. Universal approximation using radial-basis-function networks. Neural computation, 1991,3: 246 _ 257

[14] Narendra KS (1991) Adaptive control using neural networks. In: Neural networks for control.MIT Press, Cambridge, MA, pp 115-142 